\title{
FUZZY AGGREGATION FOR RANKING IN BUSINESS
}

\author{
Eva Rakovská ${ }^{1}$
}

\begin{abstract}
Today, businesses depend strongly on data and the opinion of customers or the experience of managers or experts. The large databases contain non-heterogeneous data, which is the ground for further decisions. Business uses multicriterial decisions in more areas (e.g., customer care, marketing, product development, risk management, HR, etc.) and often it is based on assessment. One of the assessment methods is the ranking, which can be done by crisp values of data where the sharp borders between evaluated entities do not give the adequate ranking result. On the other hand, the ranking process is based on the qualitative assessment, which has linguistic expression. It is more familiar and understandable for people. The article shows how to treat non-heterogeneous data to prepare them for a ranking process using fuzzy sets theory. The article aims at offering several types of ranking methods based on different inputs and preferences of the user and describes appropriate fuzzy aggregations for solving the ranking problem.
\end{abstract}

JEL Classification Numbers: C65, C18, DOI: https://doi.org/10.12955/peb.v2.258

Keywords: assessment, ranking, fuzzy aggregation, knowledge discovery in databases

\section{Introduction}

Today, businesses depend strongly on data and the opinion of customers, managers, experts, and all stakeholders. The large databases consist of many data, which hide much useful information and knowledge for managerial decisions concerning all business processes. Knowledge data discovery is necessary for informed decisions, so here are plenty of methods of how to find the relevant information and knowledge within data. The classic methods of Business Intelligence, which are based on database techniques (e.g., Online Analytic Processing - OLAP), were sufficient a few years ago, but the large size of databases and the variety of data does not allow to use of these classic methods effectively. Therefore it is necessary to use newer and faster methods, which allow automating the knowledge discovery processes with all outputs in an understandable form for all users. Machine learning methods allow finding patterns and relationships within data quickly using combinations of various mathematic models and learning from training data. It leads to automatization, but sometimes we need to solve another kind of task as the classification or prediction is. Although classification, clustering, and prediction are the core of many other tasks, the managerial processes also deal with tasks like planning, scheduling, assessment, monitoring, assignment, various types of designing. These tasks are so-called knowledgeoriented tasks Schreiber et al. (1999), where the knowledge "how to do it" plays the key role and depends on the experts' experience. The assessment tasks are very often used in daily routine in business (assessment of processes, employees, products, services, customers, projects, etc.), and it comes from knowledge of evaluators and data. Data can be acquired from surveys or databases and describes various features of evaluated items.

It is not possible to strictly say what assessment means, but Cambridge dictionary (2021) says that assessment is in a general sense:

1. "The act of judging or deciding the amount, value, quality, or importance of something, or the judgment or decision that is made"

2. "The process of considering all the information about a situation or a person and making a judgement".

3. "The process of considering the amount or value of something, or the decision that is made ".

Usually, the term assessment is connected with learning, and the assessment is ,the process of testing, and making a judgment about, someone's knowledge, ability, skills, etc. (in teaching and human resource)“ defined Cambridge dictionary (2021). Or by Bhasin (2008) "Assessment is defined as a proper collection, interpretation, and use of information in regards to learning. It gives the teacher a better awareness about the knowledge of peoples and their understanding and what are learning experiences are also about their skills and personal characters and capabilities" Bhasin (2008). When we consider the general description of assessment, it is a core of many decisions in business. It is clear, that the universal method of assessment does not exist. We know ten basic types of assessment in the human resource Bhasin (2008), but other tasks are how to assess products, stuff, processes, etc., within the enterprise. Or how to do customers` evaluation of products or services. It is possible to use various

\footnotetext{
${ }^{1}$ University of Economics in Bratislava, Faculty of Economic Informatics, Department of Applied Informatics, Bratislava, Slovakia, eva.rakovska@euba.sk
} 
decision-making methods to evaluate these tasks, but multi-criteria decision-making methods are most often used.

One of the multicriteria decision-making methods is ranking. The ranking is a process „of giving a specified rank to someone or something" Lexico (2021) or Cambridge dictionary (2021) writes it is ,a list that compares the importance, quality, success, etc. of people or things of the same type " and „the action of deciding where someone or something should be in a list that compares things of the same type". From a mathematical view, ranking is the process how to order a set of items based on some features or criteria. In this case, we cannot speak about the total ordered set but only about weak order or total preorder of items. The reason is simple: the features are not measurable, and sometimes we deal with the ordinal data or nominal data as features. The value of features can often not be expressed by numerical data or exact „strings“ without sense. The features involve the values that have a different sense for different people (for example, every person perceives the colors in a different way, the sentence „it is raining" means for somebody ,the rain is pouring" for another person „,it is raining lightly"). Or they can be burdened by the uncertainty that comes from linguistic expression. In practice, the ranking can give us the relevant picture about our business and help us by classifying some objects and items. We know, for example, the ranking of universities, banks, various institutions, websites, e-shops, products, services in the same area, etc. Then thanks to the ranking, we are able to make well-informed decisions.

The importance of ranking in business leads us to consider a generalization of ranking types and how to treat various types of data, which describes the attributes and characteristics of ranking objects. Motivation starts from the project (mentioned in Acknowledgement). Rakovská\&Hudec (2020) suggest the relevant fuzzy model, which allows to evaluated users`opinion with the software used within a company. The main idea of evaluation is to rank the users` opinion using the same questionary for each group of users and then evaluate it appropriately. The basic approach, which used fuzzy rules system (intuitive decision tree), failed because of too many rules and inability to count with missing values, with the different number of questions for each group and different answer granularity. The three-leveled fuzzy aggregation model Rakovská\&Hudec (2019) includes three types of fuzzy aggregation for a very complex model of evaluation. In practice, we can see many types of evaluations (assessment) which have the output of weakly ordered objects as we can see in the Rakovská\&Hudec (2019) and Rakovská\&Hudec (2020). The main aim of the article is to suggest some models of ranking types and show which type of aggregation is appropriate in each type of ranking model. The remainder of the paper is organized as follows: the next section describes how to transform the data to values from unit intervals and so prepare it for further counting using fuzzy aggregation functions. Then continues the section, which deals with some types of fuzzy aggregation functions and how to use them in some specific cases.

\section{Data transformation to use fuzzy aggregation}

As we mentioned before, the ranking needs data, which is usually gained from questionnaires and is stored in the databases. The process of storing the data runs automatically and fluently; data comes from distributed information systems and web applications. Generally, the ranking handles two main issues: (i) the variety of data and (ii) the number and quality of evaluators.

The data as properties` values, is which are usually heterogeneous (numbers - e.g., a number of students; binary values -"yes-no" answer; linguistic values- if the evaluator agrees with the property "agree strong, do not agree, etc..." or if a property is important, how much it is important, etc.) Sometimes the problem is that the properties do not play the same role by evaluation (some could be mandatory and others optional, or the importance of each property could be different). Also, some values of properties have not to be filled, so the ranking model should be able to count with missing values. As Hudec\& Vucetic (2019) write this is the problem of a "variety feature" of big data.

The second issue is concerning the number and quality of evaluators. The experts involved in the process of ranking should consider various aspects and know the domain area very well. But they can have different experiences, and so the ranking is very subjective. Sometimes, it is better to evaluate more experts and then to the final aggregate ranking.

The information system can be perceived as a rough set Skowron\&Dutta (2018) "Suppose we are given a pair $I S=(U, A T)$ of non-empty, finite sets $\mathrm{U}$ and AT, where $\mathrm{U}$ is the universe of objects, and AT is a 
set consisting of attributes. Each attribute can be considered as a function at:U $\rightarrow V a t$, where Vat is the set of values for the attribute $a t$, called the domain of $a t$. The pair $I S=(U, A T)$ is called an information system" and the database can be represented Skowron (2015) as $T p=(U p, A p)$, where $U p$ is a universe of entities and $A p$ is a set of attributes in a table $T p, p=1, \ldots, n$. The table contains rows that are labeled by entities (e.g., products for ranking) and columns by attributes (e.g., various attributes of products).

Considering the former two issues heterogeneity of data and more evaluators, Figure 1 describes the problem of ranking, where are more Evaluators (number $k$ ), and the evaluators' rate $m$ products $(P i$, $i=1 \ldots m)$ by using the set of products`attributes $(A j, j=1 \ldots n)$. As was mentioned former, the data can be heterogeneous; therefore it is necessary to transform it into a uniform type.

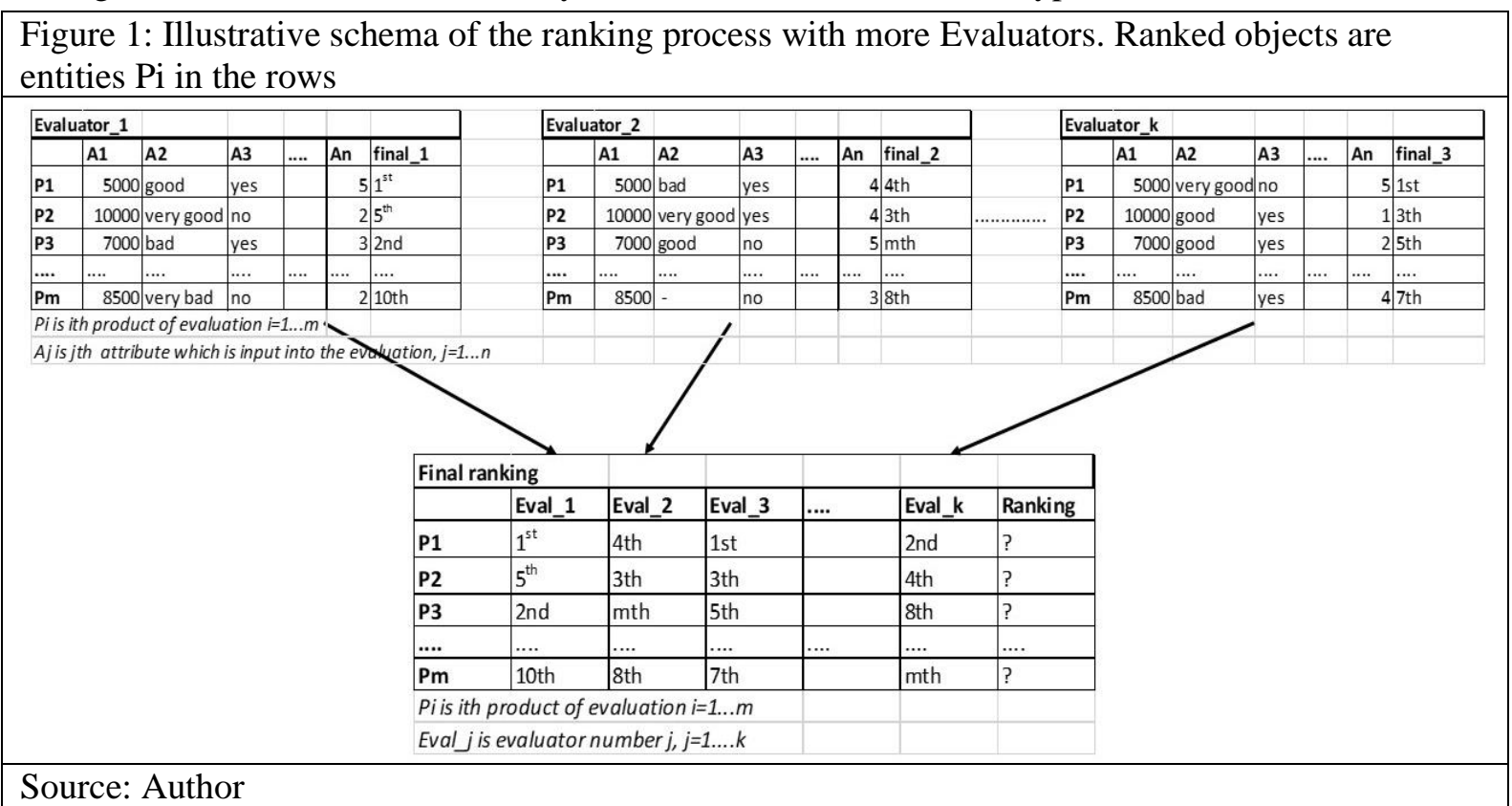

Changing the ranking to flexible evaluation is possible by using fuzzy sets and the membership function of the set. A fuzzy set $\mathrm{F}$ over the universe of discourse $\mathrm{X}$ is defined by the membership function $\mu_{\mathrm{F}}$ that matches each element of $X$ with its degree of membership to the set $F$ (Zadeh, 1965)

$\mu_{F}(x): X \rightarrow[0,1]$

where $\mu_{\mathrm{F}}(\mathrm{x})=0$ means that an element $\mathrm{x}$ does not belong to $\mathrm{F}$, while $\mu_{\mathrm{F}}(\mathrm{x})=1$ means that $\mathrm{x}$ is a full member of $F$. A value $\left.\mu_{\mathrm{F}}(\mathrm{x}) \in\right] 0 ; 1[$ indicates the intensity by which the element $\mathrm{x}$ belongs to $\mathrm{F}$. An example is set $\mathrm{X}$, which means high opinion. The maximal rating score means clear belonging to this set, whereas the significant rating belongs with a slightly lower degree. When the universal set $\mathrm{X}$ contains few elements (e.g., the scale of possible answers), we directly assign a matching degree to each element Rakovská\&Hudec (2020). Usually, people are familiar with linguistic expression e.g., like it very much, much, average, not much. So it is natural to use a linguistic approach for representing qualitative aspects of ranking. Often qualitative aspect is expressed by the Likert scale found in Likert (1932), which correspondents to linguistic terms with various granularity. The granularity is usually represented by the scale of an odd number of digits because it captures the fact that human beings can reasonably manage to bear in mind seven or so items, as write Herrera\&Martinez (2001). Figure 2 describes the representation of categorical answers and their mapping into the interval $[0,10]$ in various granularity. These scales can be straightforwardly transformed into the unit interval if it is required. Still, such linguistic terms are discrete and not in all cases is suitable to treat with discrete values. Therefore Herrera\&Martinez (2001) use another approach, the 2-tuple fuzzy linguistic representation model, which carries out processes of "computing with words" easily and without loss of information, and the linguistic domain is continuous.

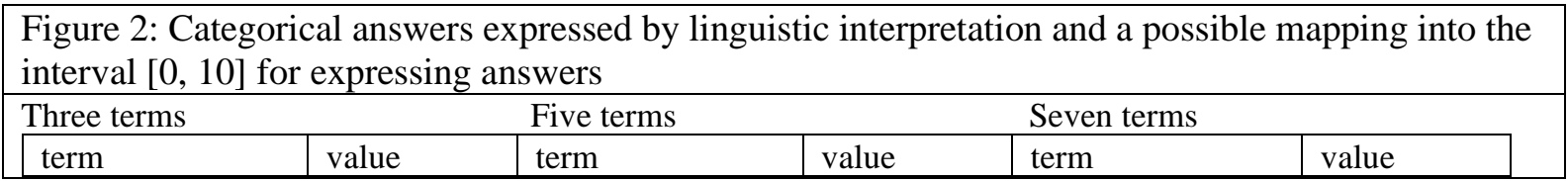




\begin{tabular}{|c|c|c|c|c|c|}
\hline $\begin{array}{l}\text { negative } \\
\text { neutral } \\
\text { positive }\end{array}$ & 9 & $\begin{array}{l}\text { very negative } \\
\text { negative } \\
\text { neutral } \\
\text { positive } \\
\text { very positive }\end{array}$ & $\begin{array}{l}1 \\
3 \\
5 \\
7 \\
7 \\
9\end{array}$ & $\begin{array}{l}\text { very negative } \\
\text { negative } \\
\text { more neut. than } \\
\text { neg. } \\
\text { neutral } \\
\text { more neut. than pos. } \\
\text { positive } \\
\text { very positive }\end{array}$ & $\begin{array}{l}0.5 \\
2 \\
3.5 \\
5 \\
6.5 \\
8 \\
9.5\end{array}$ \\
\hline
\end{tabular}

The other types of data are easily transformable to the fuzzy approach described by Zadeh (1965), for example, the number of students at a university, we can express using the membership function of the fuzzy set high number of students and binary data type, which represent naturally 0 and 1 (or true-false, yes-no) still we can keep in the domain of membership function $[0,1]$.

Then the ranking task introduces the problem of weak ordering the entities P1 to Pm (for one evaluator) by attributes in Table 1 , where the $\operatorname{Vij} i=1 \ldots . n, j=1 \ldots . m$ are values of attributes from unit interval. The main task of ranking is how to aggregate all values of attributes for each entity appropriately and finally achieve a weak ordering of all entities Pi. Here is a difference between sorting and ranking. As Pey (2015) writes, "Sorting is the action of giving an order to a set of items using an explicit set of values present among the attributes of the elements being sorted. Ranking is applying an algorithm that gives order based on different heuristics or metrics that determine importance or relevance comparing the elements in the dataset ranked". Therefore finding the right algorithm for ranking entities means finding an adequate mathematical model. We introduce the possibility of aggregating the fuzzified values. How to create aggregation depends on (i) the type of data (ii) the preferences by evaluating (whether some attributes are mandatory or optional, whether some attributes are more important by an evaluation).

Table 1: Table with entities $(\mathrm{Pi}, \mathrm{i}=1 \ldots \mathrm{m})$ for ranking by attributes $(\mathrm{Aj}, \mathrm{j}=1 \ldots \mathrm{n})$ with transformed values into the unit interval

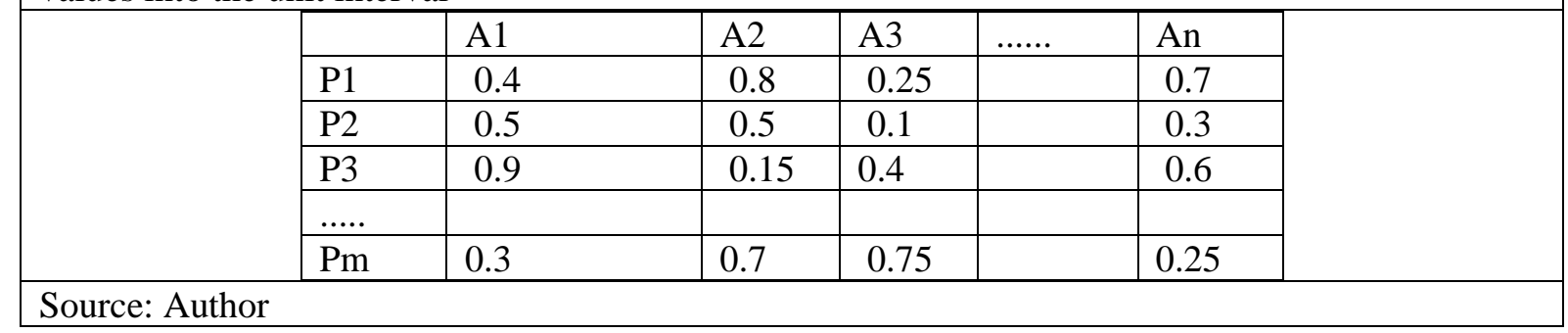

\section{Fuzzy aggregation of ranking tasks}

Coming from this basic task of how to rank the entities according to attributes by one person and considering vague, incomplete values, it is necessary to deal with fuzzy sets and fuzzy numbers. To gain the ranking of all entities, we assume to use fuzzy aggregation Beliakov et al. (2007). First, across one row (the final value for one entity), to get the value from unit interval as a result that expresses the value for further ranking of all entities. The choice of the fuzzy aggregation depends strongly on the type of the values and the evaluator' preferences (what is important at evaluation, which attributes are most important and which less etc.). In a case, when we have more evaluators, it is necessary to aggregate the gained values for each entity from all evaluators in Figure 1 which means to use the appropriate type of aggregation. First, we suppose to transform the data to a uniform type e.g., to fuzzify all values Vij, and then use the appropriate class of fuzzy aggregation. Beliakov (2009) are mentioned further fuzzy aggregation function classes, coming from the basic definition of fuzzy aggregation function.

- Averaging functions (if it is bounded by $\min (x)=\min _{i=1 \ldots n} x i \leq f(x) \leq \max _{i=1 \ldots n} x i=\max (x)$ ) e.g. weighted arithemtic means, weigthed quasi artithmetic means, ordered weighted averaging (OWA), generalised OWA and other means, medians or fuzzy integrals

- Conjunctive functions (if it is bounded by $f(x) \leq \min (x)$ and disjunctive if it is bounded by $\max (x)$ $\leq f(x)$ ), e.g., triangular norms and conorms, various types of copulas and their duals, weighted $\mathrm{t}$-norms and t-conorms or functions defined pointwise 
- Mixed if it is neither conjunctive, disjunctive or averaging e.g. uninorms, nullnorms, generated functions with neutral element, weighted generated uninorms, T-S functions, and T-OWA, SOWA, ST-OWA functions, etc.

- Idempotent if $f(t, t, \ldots, t)=t$ for any $t \in[0,1]$. Monotonicity and idempotency implies averaging behaviour

- Symmetric (commutative) if $f(x)=f\left(x_{P}\right)$ for any $x \in[0,1]^{\mathrm{n}}$ and any permutation $\mathrm{P}$ of $\{1, \ldots$, n\}

The problem of how to choose an appropriate fuzzy aggregation function in practice is not easy and to solve this problem is necessary to have much experience and knowledge. The choice depends on the mathematical attributes of the data. So in the next, we try to describe some typical cases of ranking problems and classify them into some categories.

As we mentioned before, the basic task of ranking the entities according to attributes by one person is in Table 1. A similar problem as the ranking problem is introduced in Rakovská\&Hudec (2019), where the authors solve the three-levelled model for evaluating software within all departments in the company. The final result is scoring the usefulness of various software within the company. This model comes from data collected through questionnaires, and the answers were collected as numbers representing the Likert scale (1932). So the evaluation of attributes for each entity (software) has the value from 1 to 5 , resp. 1 to 7 or to 10 . Although the answers do not belong to the unit interval, they might be straightforwardly converted to this interval. Rakovská\&Hudec (2019) have the presumption, that all values are filled, and so suitable aggregation is average aggregation and due to counting with numbers authors use the arithmetic mean due to full neutrality and effectivity (i.e., low values are fully compensated by high ones). This is a special case when we do not need to handle non-heterogenous data. The model, in the Table 1 expects that all values are transformed to values from unit interval by using the membership function of fuzzy sets. In such a case, we can take some types of ranking

- When all attributes are mandatory, we can use geometric means Beliakov, (2009). A geometric mean is a simple multiplicative scoring (all inputs are mandatory regardless of their importance). The geometric mean provides the result between the best and worst evaluation for all cases, whereas uni-norms provide such a solution only for entities that are badly evaluated in several attributes and positively in the other ones.

- The second, when all attributes are optional, and anyone is preferred, so it means the most of attributes should be satisfied in Sojka et al. (2020)

- The third, when some attributes are mandatory, and others are optional in Sojka et al. (2020)

- The fourth, when we speak about „nested queries“ and the result of the finding nested queries within the database is a list of entities that fully or partially meet the condition ranked downward from the best by the intensity of matching degrees in Sojka et al. (2020)

The last three cases are described in Sojka et al. (2020), where authors count quantified summaries of predicates in databases. The predicates express the attributes, which have an impact on further decisions. The final solution of quantified summaries is a value that represents the validity or truth value of the evaluated quantified sentence expressed within the unit interval, which allows us to compare the values for ranking entities by one evaluator.

So, in the second case, we assume that all attributes are connected by the conjunction and are optional; we can count the aggregation the same way as Sojka et al. (2020). To avoid the problems as "empty answer values" and "evaluating by the number of attributes" that are better (the entity with more attributes with better values are preferred in ranking) we replace the conjunction with relative quantifier: most of the attributes should be satisfied. Then appropriate aggregation is based on quantified summaries Sojka et al.(2020)

$v(x)=\mu_{Q}\left(\frac{1}{n} \sum_{i=1}^{n} \mu_{p i}(x)\right)$

where $\mathrm{n}$ is the number of atomic predicates (attributes) posed on a subset of attributes in the database, $y=\frac{1}{n} \sum_{i=1}^{n} \mu_{p i}(x)$ is the proportion of atomic predicates pi that are satisfied by the entity $\mathrm{x}$ being evaluated and $\mu_{\mathrm{Q}}$ is the formalization of the quantifier most of. The truth value $\mathrm{v}$ assumes values from the unit interval. 
The third case represents the situation, where some attributes are mandatory and others are optional. To covered these requirements Sojka et al.(2020) modified (2) and used conjunction in the fuzzy logic, especially the minimum t-norms in Beliakov et al (2007) and quantified summary in such case is expressed by

$v(x)=\left(\bigwedge_{i=1}^{r} P_{i}(x)\right) \wedge\left(\mu_{Q}\left(\frac{1}{s} \sum_{j=1}^{s} \mu_{p j}(x)\right)\right.$

In the first three cases described previously, the solution of aggregation is the score for every entity (Pi in Table 1) from one evaluator, and the value is within the unit interval. A further question is how to aggregate acquired values for each entity $\mathrm{Pi}, \mathrm{i}=1 \ldots . \mathrm{n}$ in the group of all evaluators. Table 2 indicates an illustrative case, where rows represent the entities, and the values were counted based on evaluation of attributes from evaluators Eval_j, $\mathrm{j}=1 \ldots 4$. The last column represents the possibility of aggregating values using arithmetic mean, which seemed suitable for this case. The values within Table 2 are in the unit interval, and it is a good assumption to use each class of aggregation Beliakov et al. (2007). Rakovská\&Hudec (2019) discussed the suitability of disjunctive, averaging, and conjunctive aggregation functions and compare them. Also, Rakovská\&Hudec (2019) compare the uni-norm, geometric, and arithmetic means regarding their specific features. Each aforementioned function gives the solution in the unit interval, where the numbers are easily ordered.

The fourth case is a specific one, where the solution is ranked list of some entities. For example, when we want to rank the universities by nested queries we can choose a ranked list of universities, which meet the condition select universities where most of the faculties are technically oriented and have a high number of women students. This case allows to make the direct ranking within the specific group from the database but is not able to compare the entities from various groups. The procedure for calculating validities is created straightforwardly as the extension of (2) Sojka et al. (2020), and the method is integrated with the vertical and horizontal aggregation Sojka et al. (2020).

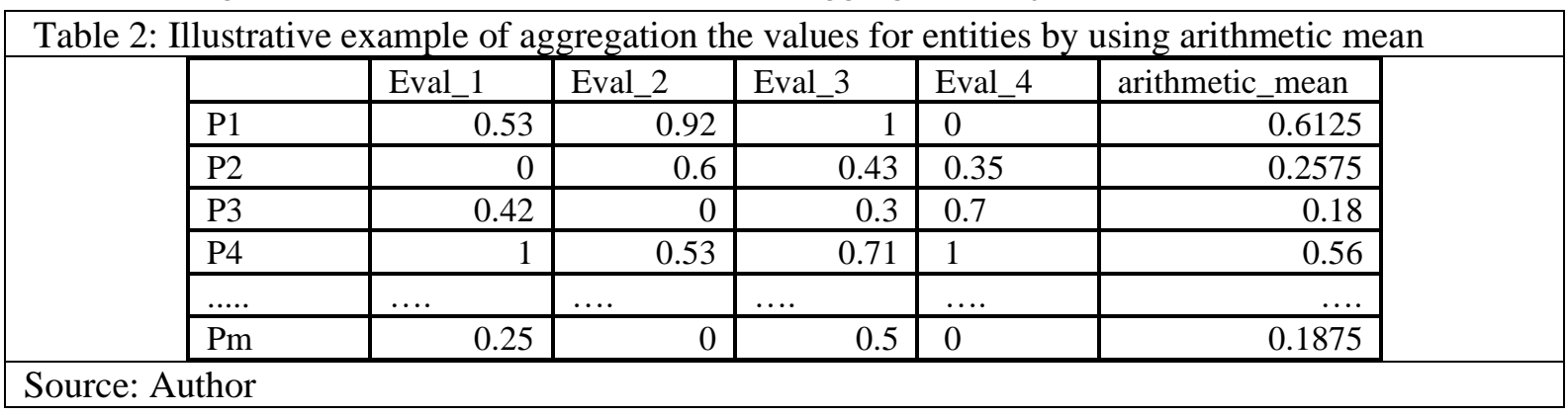

\section{Conclusion}

This article is prepared as a part of the project "Big data analytics as a tool for increasing the competitiveness of enterprises and supporting informed decisions", where Big data is core for finding some patterns, which are important to design a decision support system model for small enterprises. It is not easy to find an appropriate decision support system for small enterprises and so one aim of the project is to design DSS for finding an appropriate logistic model comparing the customer preferences with a large amount of data in the database. The ranking process allows the possibility to make wellinformed decisions on which logistic model best fits a specific case and allows to classify various cases. The fuzzy ranking process seems to be easily usable in practice, because it catches the qualitative aspect of decisions using linguistic terms and brings flexibility into the decision process. It avoids the problem of empty answers and allows to treat with variability of preferences (mandatory, optional, group of entities with mandatory and optional attributes, etc.). As mentioned before, the classes of fuzzy aggregation functions contain many various functions. It is not trivial to choose the right function for a real problem because it is necessary to explore the mathematical properties of the examined problem. However, future research will be a focus on conformance measures Hudec\&Vucetic (2019) and their application in the ranking problem. The task is to measure the difference between aggregated values from more evaluators for the same entity and then order values by this difference. 


\section{Acknowledgments}

This work is supported by a project VEGA No. 1/0373/18 entitled "Big data analytics as a tool for increasing the competitiveness of enterprises and supporting informed decisions" by the Ministry of Education, Science, Research and Sport of the Slovak Republic.

\section{References}

Beliakov, G., Pradera, A., \& Calvo S. T. (2007). Aggregation Functions: A Guide for Practitioners. Springer-Verlag,

Berlin, Heidelberg

Beliakov, G. (2009, July) Introduction to fuzzy systems and aggregation operators. [Web presentation post]. Retrieved from https://iemae.upc.edu/ca/seminari-del-institut/realitzats/conf.beliakov.22.07.09

Bhasin, H. (2008, December 22) Top 10 Types of Assessment. [Web article]. Retrieved from https://www.marketing91.com/types-of-assessment/

Cambridge Dictionary (2021). Assessment. Ranking. [Web dictionary post] Retrieved from https://dictionary.cambridge.org/dictionary/english

Herrera, F., \& Martinez, L. (2001). A model based on linguistic 2-tuples for dealing with multi granular hierarchical linguistic contexts in multi-expert decision-making. IEEE Transactions on Systems, Man and Cybernetics, Part B (Cybernetics), 31(2), 227-234. https://doi.org/10.1109/3477.915345

Hudec, M., \& Vučetić Miljan. (2019). Aggregation of Fuzzy Conformances. New Trends in Aggregation Theory, 302-314 https://doi.org/10.1007/978-3-030-19494-9_28

Lexico Oxford Dictionary (2021). Ranking. [Web dictionary post] Retrieved from https://www.lexico.com/definition/ranking

Likert, R. (1932). A technique for the measurement of attitudes, Archives of Psychology 22(140): 1-55

Pey, J.L.L. (2015, November 6). What are the differences between Sorting and Ranking? [Web blog post]. Retrieved from https://www.quora.com/What-are-the-differences-between-Sorting-and-Ranking

Rakovská, E., \& Hudec, M. (2019). A Three-Level Aggregation Model for Evaluating Software Usability by Fuzzy Logic. International Journal of Applied Mathematics and Computer Science, 29(3), 489-501. https://doi.org/10.2478/amcs2019-0036

Rakovská, E., \&amp; Hudec, M. (2019). Two Approaches for the Computational Model for Software Usability in Practice. Advances in Intelligent Systems and Computing, 191-202. https://doi.org/10.1007/978-3-030-18058-4_15

Schreiber, G., Akkermans, H., Anjewierden, A., de Hoog, R., Shadbolt, N. R., Van de Velde, W., \& Wielinga, B. J. (1999) Knowledge Engineering and Management. https://doi.org/10.7551/mitpress/4073.001.0001

Skowron, A., Jankowski, A., \& Swiniarski, R. W. (2015). Foundations of Rough Sets. Springer Handbook of Computational Intelligence, 331-348. https://doi.org/10.1007/978-3-662-43505-2_21

Skowron, A., \& Dutta, S. (2018). Rough sets: past, present, and future. Natural Computing, 17(4), 855-876. https://doi.org/10.1007/s11047-018-9700-3

Sojka, P., Hudec, M., \& Švaňa, M. (2020). Linguistic Summaries in Evaluating Elementary Conditions, Summarizing Data and Managing Nested Queries. Informatica, 1-16. https://doi.org/10.15388/20-infor428

Zadeh, L.A. (1965). Fuzzy sets. Information and Control, 8, 338-353 https://doi.org/10.1016/S0019-9958(65)90241-X 